\title{
AIDS Enters New Era with Pharmacists on the Front Lines
}
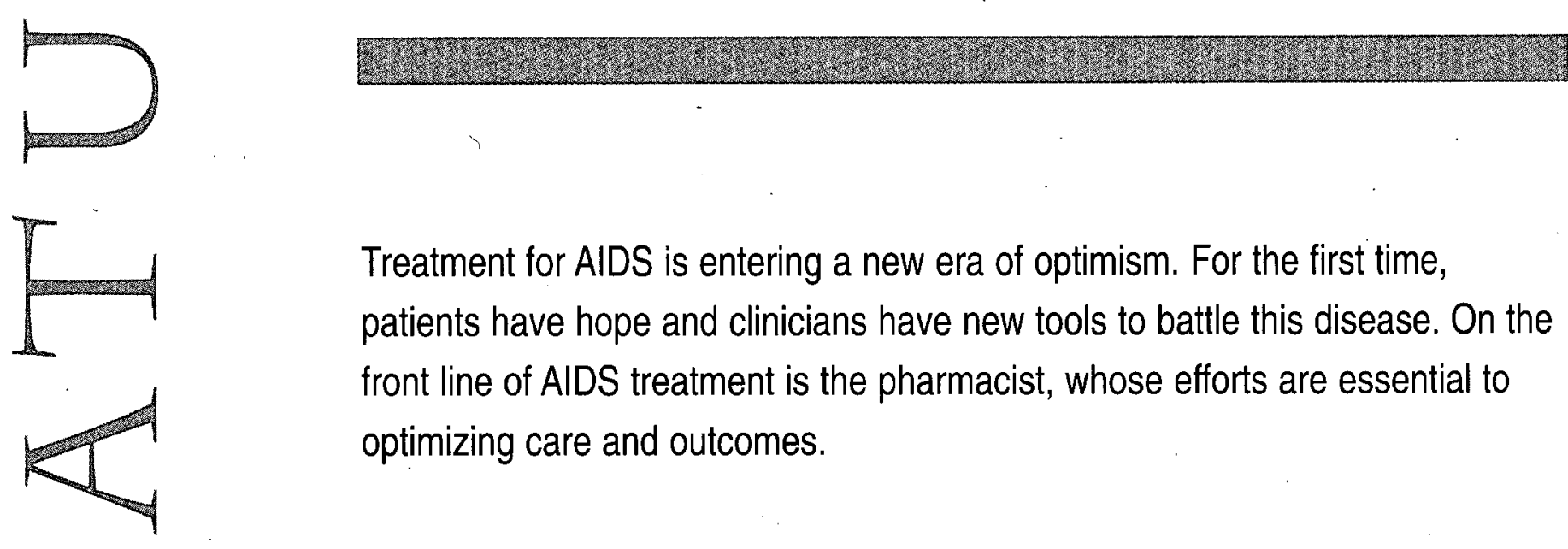

Treatment for AIDS is entering a new era of optimism. For the first time, patients have hope and clinicians have new tools to battle this disease. On the front line of AIDS treatment is the pharmacist, whose efforts are essential to optimizing care and outcomes.
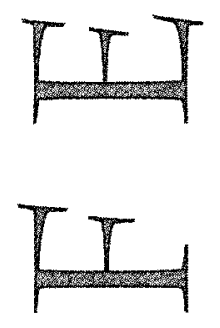

\footnotetext{
Ine years ago, a diagnosis of AIDS

1 was as good as a death sentence.

1 Today, many AIDS patients are

doing something they only could have dreamt of previously-they are planning for the future. At the same time; clinicians caring for AIDS patients are beginning to treat it as a chronic condition instead of a terminal illness and are willing to face the challenges of this changing treatment paradigm. What is behind this newfound optimism? The use of drug "cocktails" with protease inhibitors and new tools to measure and track viral load take much of the credit. Thanks largely to these innovations, there is new hope for AIDS patients.

Three educational sessions at the Academy of Managed Care Pharmacy's Ninth Annual Meeting in New Orleans addressed the treatment of HIV/AIDS and the role of pharmacists in treating AIDS patients. This article summarizes the highlights of these presentations.
}

\section{J OANNE. KALDY}

Joanne Kaldy is Managing Editor of the Journal of Managed Care Pharmacy.

Copyright (C 1997, Academy of Managed Care Pharmacy, Inc. All rights reserved. 


\section{INNOVATIONS IN DRUG THERAPY}

Until 1995, nucleoside reverse transcriptase inhibitors (RTIs) such as AZT and ddI were state-of-the-art medications for treating AIDS. While they helped inhibit replication of AIDS cells, the development of protease inhibitors took treatment to a new level. Protease inhibitors are antiviral compounds that chemically inhibit HIV reproduction by disabling the enzyme protease. As Gordon Vanscoy, vice president, Clinical Care Division of Stadtlanders Managed Pharmacy Services and assistant dean for managed care, University of Pittsburgh, said, "When protease inhibitors hit the market, we had patients who were prepared to die and then were confronted with life."

Currently, there are four protease inhibitors on the market: saquinavir, ritonavir, indinavir, and nelfinavir. Two additional compounds, 1W1 W94 and ABT-378, are still in trial. Protease inhibitors show promising results when used in combination with nucleoside analogues. Dr. Vanscoy noted that for the first time since the first case of AIDS was diagnosed in the early 1980s, deaths from the disease declined last year. At the same time, some studies have linked new therapies with reduced lengths of hospital stays as well as reductions in hospitalizations. However, Vanscoy pointed out that there are some complicating issues. For example, he noted, the cost of drug therapy has risen to $\$ 12,000-\$ 15,000$ per year for AIDS patients taking drug therapy combinations that include protease inhibitors. There are still many questions about what therapy combinations work best for which patients. "The complexity of managing AIDS patients has gone up tremendously," said Vanscoy, and this has implications for pharmacists. "The standards of practice [for treating AIDS patients] change two to three times a year." This presents a challenge to pharmacists and other practitioners to remain current with the literature and technology.

\section{NEW TECHNOLOGIES ADD TO PRACTICE STANDARDS}

"We now have technology to more completely understand AIDS," said Timothy Schacker, M.D., assistant professor of medicine, Division of Infectious Diseases, University of Minnesota. "We now can take and measure HIV in the blood and get results back in a few days. This is a major revolution. We're actually measuring the amount of replicating virus in patients' blood." This HIV RNA test is "an accurate prediction of who will do well and who will do poorly," he noted. "We now can go into the lymph node and actually detect how

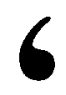

\section{It is important for clinicians to determine whether their} goal is to suppress or eradicate the disease.

\section{- Timothy Schacker \\ ,}

much virus is there." Schacker observed that this enables clinicians to understand the 'quantity' of the illness, or viral load, in a particular patient.

Protease inhibitors prevent the assembly of viral particles. When used in various combinations with other drugs, they have been shown to reduce viral loads significantly. What impact do the various combinations of nucleosides, nonnucleosides, and protease inhibitors have, and what combinations work best? The jury is still out on this, Schacker says, noting that studies to provide some answers currently are underway.

It is important, Schacker insisted, for clinicians to determine whether their goal is to suppress or eradicate the dis- ease. Under the suppression model, goals would include effectively suppressing replication, delaying emergence of resistance to treatment, and prolonging disease-free intervals. The principles of eradication include: preventing resistance with complete suppression, eliminating sanctions, and designing regimens to cover genetic variants. The treatment strategy here is "treat early, treat hard."

There is much controversy about the right goals, Schacker said. "If you believe in the suppression model, you have agreed that you won't cure, but you'll help the patient live a long, productive life without symptoms and with a minimum of medications," adding, "Pharmacists have an obligation to stay current and add value to the care paradigm." Increasingly, AIDS patients are beginning to manage their medications the way they do for chronic illnesses, according to Vanscoy. "They feel better, and they stop taking their medication." This is of particular concern, as compliance is essential to the effectiveness of protease inhibitor therapy; noncompliance can lead to resistance to treatment.

\section{EVALUATING RESPONSE TO THERAPY}

Quantitative measures of HIV are now available to monitor response to antiretroviral therapy and the progression of AIDS. Viral load is the generic term that describes the results of one of several assays used in clinical laboratories. "Plasma HIV RNA peaks rapidly in the days following initial infection and then falls to a level that is maintained for a long period of time," said Rebecca Coleman, Pharm.D., research pharmacist, San Francisco General Hospital. "Disease progression is marked by an increase in plasma RNA levels, resulting in very high levels in late disease. Plotted across time, plasma HIV RNA appears to vary in a fashion converse to CD4 count." Plasma HIV RNA levels can be used as direct measures of drug efficacy.

There are several issues that must 
Continued from page 392

be addressed regarding viral load assays, said Coleman. For one, individuals responsible for working with specimens should fully understand proper handling procedures. It also is important to understand the lower limit of detection. Coleman outlined several recommendations for taking viral loads, including two baseline samples at least three days apart; within one month following change in therapy; every three months.

Recommendations for using viral load testing in clinical practice include:

A Baseline values should be established using two tests conducted 2-4 weeks apart. Routine monitoring should be conducted every 3-4 months.

A Additional tests should be done at critical decision points and 3-4 weeks following initiation or change in therapy.

A Testing should not be undertaken within a month of acute illness or vaccination.

A Whenever possible, all viral load testing for a single patient should be conducted by the same lab to minimize variability.

$\Delta$. Changes greater than three-fold or 0.5 log can be considered "real' as they exceed the variation known to be inherent in these assays when used in the clinical setting.

\section{CHALLENGES OF DRUG THERAPY}

"There's more debate than ever about when to initiate therapy," said Coleman. And the choice of drugs only will become more complex in the rear future. "Changing therapy is mostly an issue of tolerance as opposed to lack of efficacy," she observed: The complexity and challenges of drug therapy for AIDS patients requires vigilance on the part of pharmacists. Since, as Coleman suggest$\mathrm{ed}$, there is no evidence to indicate that drug therapy can be stopped at any . time, the pharmacist can expect to make a long-term commitment to working with these patients.

Lidia Gajewski, Pharm.D., manager, Managed Clinical Care Division, Stadtlanders Managed Phärmacy Services, Pittsburgh, agreed that the development of protease inhibitors has changed the way AIDS is treated and increased the pharmacist's role in caring for AIDS patients. However, she warned that "our nemesis is likely to be resistance. Resistance occurs subsequent to mutations on the protease gene. The emergence of resistance requires viral replication in the presence of inhibitors," and there is a substantial risk of cross-resistance in this class. Factors that favor resistance include: subtherapeutic drug concentra-

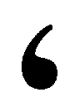

Pharmacists can and do play. a role in managing the care of AIDS patients. And we can make a difference.

\section{-Rebecca Coleman}

\section{9}

tions (including suboptimal dosing, drug interactions, noncompliance, and the effects of food) and monotherapy.

According to Coleman, it is important to consider the potential clinical consequences of resistance which include disease progression, limited therapeutic options, and the potential transmission of resistant HIV. The pharmacist can play an important role in monitoring and preventing drug interactions, she noted, adding that the potential for such problems with this class of drugs is "tremendous."

"Patients on protease inhibitors are dealing with many issues that may make it difficult for them to comply with treatment," said Coleman, "but compliance is essential." Studies have found a relationship between noncompliance and increased viral load. A minor dropoff in compliance potentially could result in resistance. Adverse drug reactions (ADRs) "can contribute to noncompliance, so we as pharmacists should work with these patients to help them with these problems," she observed.

How can pharmacists help prevent resistance? One important guiding principle, Coleman said, is, "never add a new drug to a failing regimen-there must be at least two new agents." Strategies to minimize risk of resistance also should include optimal therapeutic dosing, preventing/monitoring for drug interactions, discouraging nonadherence and 'drug holidays,' preventing ADRs, and avoiding protease inhibitor monotherapy. "Pharmacists can and do play a role in managing the care of AIDS patients," Coleman noted. "And we can make a difference."

\section{PHARMACIST'S ROLE IN TREATING AIDS}

Clearly, pharmacists have never had a more important role in managing the care of AIDS patients. In fact, it is fair to say that the pharmacist's active involvement is essential. As Dr. Schacker noted, "In terms of HIV/AIDS management, you play a critical role in the successful management of these patients."

There are several pharmacy-related issues particular to AIDS, said Leslie Fish, Pharm.D., clinical pharmacologist, Fallon Clinic-Division of Drug Utilization, Worcester, Massachusetts, in her session. These include: lack of information regarding medications; when to start therapy; monotherapy versus combination therapy; resistance; cost factors; polypharmacy in the AIDS patient; and when to add or change therapy.

Dr. Fish noted that monotherapy is rarely used anymore; but not all combinations work, either. The pharmacist, however, iš in a key position to monitor therapy and document what does and doesn't work. When therapy doesn't work, the pharmacist needs to determine if the patient is intolerant or noncompliant. If 
noncompliance is an issue, the pharmacist should find out why. Is the patient experiencing adverse effects? Is medication cost a factor? Since resistance is a concern, it is important to determine why AIDS drug therapy isn't working and make appropriate adjustments to increase the chance of success. "You want to change therapy before the clinical progression of the disease state," Fish said, adding, "The pharmacist has a role in suggesting changes early on."

According to Fish, the pharmacist also has a role in determining what other medications patients may be taking. In 1991, the average AIDS patient was taking six prescription drugs per month, as well as an unknown quantity of over-the-counter medications. "So there's a lot pharmacists can do in this setting," she said. "We are the drug experts; we need to think of ourselves this way." Areas where pharmacists can have an impact include interviewing patients about past medications history, use of over-the-counter preparations, and drug resistance problems, along with helping to improve compliance.

Pharmacists should work closely with AIDS patients on compliance. If a patient forgets his or her doses of medication, it could seriously jeopardize the condition and lead to resistance to treatment. At the same time, the complicated dosing schedules that accompany AIDS drug therapy, as well as the high costs of these drugs, make compliance difficult for many patients.

Christine Suh, Pharm.D., pharmacist specialist/infectious diseases, Kaiser Permanente, Harbor City, California, agrees that the pharmacist has an important role to play in AIDS patient care. She described the role of the pharmacist in inpatient care to include: daily rounding of HIV-infected patients; handling drug dosing; providing patient education during hospitalization and at discharge; and facilitating continuity of care at time of discharge.

Suh defined the pharmacist's outpatient role to include: coordinating outpatient intravenous therapy; coordinating IV access, drug delivery, and appointments; and handling patient education and follow up.

Suh suggested that the pharmacist has an important role to play in monitoring for and managing intolerance. "I spend a lot of time looking at intolerance," she noted. She indicated that it is important to note when values were taken and how long the patient had been on the therapy at the time. "I make sure that antiretroviral history and viral load information is updated before the physician sees the patient," Suh said, suggesting that the pharmacist can handle these types of activities to streamline care and save time for the physician and other members of the care team.

According to Suh, the pharmacist also can be active in clinical trials by developing investigational drug protocols, completing case management forms, managing study drugs, making clinic study visits, and reporting adverse events. Fish added that the pharmacist has a role as researcher. "You have access to new drug therapies and medications. You keep these records and can look at resistance and tolerance patterns. We need to do more of this."

All of the speakers addressing HIV/AIDS at the AMCP annual meeting agreed that the pharmacist has a vital role to play in managing patients with this disease. Suh spoke about the importance of the pharmacist specialist position in infectious diseases. This individual, she said, "participates in the maintenance of the patient's health by providing the perspective and clinical knowledge required to ensure safe and effective drug therapy. Pharmacists can have an even greater impact on the health of patients in the managed care setting."

The pharmacist's role in AIDS patient care is much more likely to grow than to diminish in the coming years, as more AIDS patients live longer and receive treatment that is more consistent with chronic care. And while the advances in AIDS treatment are promising, Schacker warned, "We're in a holiday now. Most patients are responding well. We don't know if this will last. It probably won't." 